\title{
Collective diffusion in charge-stabilized suspensions: Concentration and salt effects
}

\author{
J. Gapinski and A. Patkowski \\ Institute of Physics, A. Mickiewicz, University, Umultowska 85, 61-614 Poznan, Poland
}

\begin{abstract}
A. J. Banchio
Facultad de Matemática, Astronomia y Física, Universidad Nacional de Córdoba, Ciudad Universitaria, 5000 Córdoba, Argentina

P. Holmqvist, G. Meier, M. P. Lettinga, and G. Nägele ${ }^{\text {a) }}$

Institut für Festkörperforschung, Forschungszentrum Jülich, 52425 Jülich, Germany
\end{abstract}

(Received 18 December 2006; accepted 16 January 2007; published online 13 March 2007)

\begin{abstract}
The authors present a joint experimental-theoretical study of collective diffusion properties in aqueous suspensions of charge-stabilized fluorinated latex spheres. Small-angle x-ray scattering and x-ray photon correlation spectroscopy have been used to explore the concentration and ionic-strength dependence of the static and short-time dynamic properties including the hydrodynamic function $H(q)$, the wave-number-dependent collective diffusion coefficient $D(q)$, and the intermediate scattering function over the entire accessible range. They show that all experimental data can be quantitatively described and explained by means of a recently developed accelerated Stokesian dynamics simulation method, in combination with a modified hydrodynamic many-body theory. In particular, the behavior of $H(q)$ for de-ionized and dense suspensions can be attributed to the influence of many-body hydrodynamics, without any need for postulating hydrodynamic screening to be present, as it was done in earlier work. Upper and lower boundaries are provided for the peak height of the hydrodynamic function and for the short-time self-diffusion coefficient over the entire range of added salt concentrations. (C) 2007 American Institute of Physics.
\end{abstract} [DOI: $10.1063 / 1.2538891]$

\section{INTRODUCTION}

Charge-stabilized suspensions are composed of mesoscaled colloidal particles or macromolecules dispersed in a low-molecular polar solvent. The importance of these soft matter systems is based on the fact that they ubiquitously occur in chemical, environmental, and food industry, and in many medical, cosmetic, and biological products. Prominent examples include proteins, polysaccharides and viruses, paint and clay particles, and well-characterized model systems consisting of spherical latex spheres. For the latter type of model systems, a considerable amount of work was done during the past years to explore and understand the dynamics of charged spheres on a microscopic basis. ${ }^{1,2}$ Diffusion and rheological properties of charge-stabilized colloidal suspensions are determined by a subtle interplay of electrosteric and hydrodynamic interparticle forces. At long to intermediate distances charge-stabilized colloidal spheres interact electrostatically by an exponentially screened Coulomb repulsion originating from the overlap of neutralizing electric double layers formed by surface-released counterions and residual or added salt ions.

Hydrodynamic interactions (HIs) are transmitted by complicated solvent flow patterns created by the moving particles. In unconfined suspensions of mobile particles HIs are very long range and decay with the interparticle distance $r$

\footnotetext{
a) Author to whom correspondence should be addressed. Electronic mail: g.naegele@fz-juelich.de
}

like $1 / r$. The inherent many-body character of the HIs in nondilute suspensions causes challenging problems to the theoretical and computer simulation studies of diffusion properties.

A salient measure of the strength of HIs with regard to short-time diffusion is given by the hydrodynamic function ${ }^{2}$

$$
H(q)=D_{s} / D_{0}+H_{d}(q)
$$

It is the sum of a wave-number-independent self-part and a $q$-dependent distinct part. Here, $D_{s}$ is the short-time selfdiffusion coefficient, and $D_{0}$ is the particle diffusion coefficient at infinite dilution. In the large $q$ limit, the distinct part vanishes and $H(q)$ becomes proportional to $D_{s}$. Without HIs, $H(q)$ is equal to 1 for all $q$. Any variation in its dependence on the scattering wave number is thus a hallmark of HIs. The hydrodynamic function can be interpreted in physical terms ${ }^{3}$ as a (reduced) generalized mean particle sedimentation velocity for a suspension subject to a weak periodic force field collinear to the wave vector $\boldsymbol{q}$ and oscillating like $\cos (\boldsymbol{q} \cdot \boldsymbol{r})$. Experimentally, $H(q)$ can be determined as a function of $q$ through combining a static with a dynamic scattering experiment by proceeding as follows: The static structure factor, $S(q)$, is measured by a static scattering experiment. In addition, the so-called short-time collective diffusion function, $D(q)$, is determined by x-ray photon correlation spectroscopy (XPCS) or dynamic laser light scattering (DLS) through a short-time extrapolation of the electric field autocorrelation function, $g^{(1)}(q, t)$, according to 


$$
D(q)=-\frac{1}{q^{2}} \lim _{t \rightarrow 0} \frac{d}{d t} \ln g^{(1)}(q, t) .
$$

The hydrodynamic function can then be extracted from these two measurements using the expression

$$
H(q)=\frac{D(q) S(q)}{D_{0}} .
$$

Therefore, the determination of $H(q)$ relies on high resolution data, both for $D(q)$ and $S(q)$. Recent studies on shorttime diffusion, where $H(q), D(q)$, or $D_{s}$ have been determined by appropriately selected scattering experiments, comprise charge-stabilized colloidal spheres, ${ }^{3-8}$ hard-spherelike suspensions, ${ }^{9-11}$ aqueous solutions of charged globular proteins, ${ }^{12}$ solutions of neutral and charged liposomes, ${ }^{13}$ dispersions of soft core-shell particles ${ }^{14}$ and of soft microgel spheres, ${ }^{15}$ and suspensions of polystyrene spheres with depletion-induced attraction. ${ }^{16}$

Experimental results ${ }^{17}$ for the hydrodynamic function of highly charged colloidal spheres at low volume fractions $\phi$ (typically smaller than 0.05) are in excellent agreement with the theoretical predictions of Nägele ${ }^{2}$ obtained using a pairwise-additivity approximation of HIs. In its simplest form, this theory accounts only for the leading far-field (i.e., Rotne-Prager) part of the hydrodynamic pair mobilities which dominates in very dilute systems kept at low salinity. For these systems, the probability of two or more spheres getting closer to each other than, typically, six particle radii is vanishingly small owing to the strong and long-range electrostatic repulsion. The far-field part of the HIs is pairwise additive and it includes terms up to $O\left(1 / r^{3}\right)$ in the inverse distance expansion. For dilute systems that are dominated by far-field HIs, $D_{s}$ is practically equal to $D_{0}$. In these systems the maximal value, $H\left(q_{m}\right)$, of $H(q)$ at the wave number $q_{m}$ is larger than 1 . The maximum increases with increasing concentration provided that the far-field HI part remains dominant.

The pairwise-additivity approximation necessarily fails for larger particle densities when many-body HIs come into play. ${ }^{2}$ In earlier experimental work, ${ }^{8,13}$ it had been concluded that HIs between charged colloidal particles are screened in the important case of dense (i.e., in the sense of strongly correlated) charge-stabilized suspensions at low salinity. This conclusion was based on the observation of experimentally extracted values of $H(q)$ and $D_{s}$ that are even smaller than for a suspension of neutral colloidal hard spheres at the same concentration. It should be recalled here that for hard spheres, $H\left(q_{m}\right)$ is smaller than 1 , and its value decreases linearly in volume fraction according to ${ }^{18}$

$$
H\left(q_{m}\right)=1-1.35 \phi .
$$

The linear density described by Eq. (4) is valid even up to the hard-sphere freezing volume fraction of $\phi \approx 0.5$.

The interpretation of the strong hydrodynamic hindrance in terms of hydrodynamic screening has caused a strong controversy since, according to theory, the screening of HIs should not occur for liquid-state suspensions of mobile spherical particles. ${ }^{19}$ Many-body HIs in mobile-sphere suspensions merely enlarge the effective suspension viscosity.
Therefore, on a coarse-grained level with resolved distances much larger than the typical interparticle distance, only the prefactor of the leading $1 / r$ part in the HIs is modified. It should be recalled here that, contrary to nonconfined systems of mobile spheres, hydrodynamic screening is present for systems including spatially fixed obstacles like in porous media. ${ }^{20}$ In such systems the momentum of flow perturbations is absorbed by fixed obstacles kept in place by external forces. Screening can also occur in the presence of fixed macroscopic boundaries such as walls or enclosing pipes. ${ }^{21,22}$ Furthermore, an algebraic screening of fluid flow perturbations is observed in the electrophoresis of colloidal polyions. $^{23}$

The experimental observation made in Refs. 8 and 13 on the hydrodynamic hindrance of short-time diffusion in lowsalinity charge-stabilized suspension stronger than the hardsphere prediction could not be explained by any theoretical method. The only theoretical method available until very recently that allowed us to predict the $H(q)$ of dense chargestabilized suspensions with strong many-body HIs had been the (zeroth-order) $\delta \gamma$ method of Beenakker and Mazur. ${ }^{19}$ This method accounts in an approximate way for many-body HIs through so-called ring diagrams. It requires $S(q)$ as the only external input. Originally, the $\delta \gamma$ method had been applied to hard-sphere suspensions only. In later work, it was used additionally to make predictions for charge-stabilized systems. ${ }^{2,5,8,24}$ However, the astonishingly small values for $H(q)$ and $D_{s}$ extracted in Refs. 8 and 13 could not be reproduced using the $\delta \gamma$ scheme. This observation had been interpreted in Ref. 8 as evidence of its failure, since it does not predict HI screening to be present. In contrast to neutral hard spheres, no computer simulation results had been available in the past to explore the apparent failure of the $\delta \gamma$ scheme for the important case of de-ionized charge-stabilized suspensions.

In a recent article $^{4}$ we have shown, in accord with the theoretical expectation, that there is no hydrodynamic screening present in low-salinity fluid systems. We have arrived at this result from combining carefully performed small-angle $\mathrm{X}$-ray scattering (SAXS) and XPCS measurements of $S(q), D(q)$, and $H(q)$ for relatively concentrated suspensions of charge-stabilized fluorinated latex spheres, with dynamic computer simulations made for a wide range of electrostatic repulsions. The simulations have been performed using a novel accelerated Stokesian dynamics (ASD) simulation code for Brownian particles, developed recently by Banchio and Brady. ${ }^{25}$ Our simulation results for $H(q)$ are the only ones available to date for charge-stabilized suspensions with significant many-body HIs. All our experimental data on $H(q)$ and on the collective diffusion function $D(q)$ can be quantitatively explained by the ASD simulations, and additionally by a modified (hybrid) version of the $\delta \gamma$ scheme. The modification consists of using the $\delta \gamma$ scheme for the $q$-dependent distinct part of $H(q)$ only, whereas more accurate simulation results are used to compute the selfdiffusion contribution $D_{s} / D_{0}$. In earlier applications of the $\delta \gamma$ method to charge-stabilized dispersions, the value of the self-part had been treated as a mere fitting parameter used to match the experimental peak height of $H(q) .{ }^{2,5,24}$ Quite inter- 
TABLE I. Parameters used in the ASD, $\delta \gamma$ theory, and Rogers-Young (RY) integral equation calculations for the low-salt (LS) and high-salt (HS) series of samples of fluorinated latex spheres. Within each series, three differently concentrated samples labeled as A, B, and C are studied. The effective colloid charge number, $Z$, is determined from ASD and RY fits of $S(q)$ to the experimental peak heights. System temperature: $T$ $=293.15 \mathrm{~K}$ corresponding to a Bjerrum length in water of $L_{B}=e^{2} /\left(e k_{B} T\right)=0.71 \mathrm{~nm}$ (for $\varepsilon=80.1$ ). Moreover, $C_{w}$ is the weight concentration of colloids and $C_{s}$ denotes the amount of added $\mathrm{NaCl}$. The particle radius used in the calculation is $a=82.5 \mathrm{~nm}$. This value can be considered as an effective core-shell radius (cf. Sec. IV A). From dynamic light scattering on diluted samples, the single-sphere diffusion coefficient is determined as $D_{0}=2.69$ $\times 10^{-8} \mathrm{~cm}^{2} / \mathrm{s}$. The screening parameter, $\kappa$, is calculated according to Eq. (6). The volume fractions $\phi$ have been obtained from matching the wave-number location of the theoretical structure factor peak to the experimental one. The structure factor of the high-salt samples is practically equal to that of neutral hard spheres. Therefore, no values of $Z$ are given for these systems.

\begin{tabular}{lcrlccccc}
\hline \hline Sample & $C_{s}(\mathrm{mM})$ & $C_{w}(\mathrm{~g} / \mathrm{l})$ & \multicolumn{1}{c}{$\phi$} & $Z_{\mathrm{RMSA}}$ & $Z_{\mathrm{RY}-\mathrm{ASD}}$ & $\kappa a$ & $H^{\text {expt }}\left(q_{m}\right)$ & $D_{s} / D_{0}(\mathrm{ASD})$ \\
\hline LS-A & 0.5 & 200 & 0.123 & 1600 & 1400 & 6.84 & 0.89 & 0.83 \\
LS-B & 0.5 & 97 & 0.0597 & 1800 & 1900 & 6.48 & 0.93 & 0.92 \\
LS-C & 0.5 & 75 & 0.0462 & 1600 & 1800 & 6.37 & 0.96 & 0.94 \\
HS-A & 50 & 231 & 0.142 & $\cdots$ & $\cdots$ & 65.3 & 0.82 & 0.74 \\
HS-B & 50 & 95 & 0.0585 & $\ldots$ & $\cdots$ & 65.3 & 0.88 & 0.89 \\
HS-C & 50 & 50 & 0.0308 & $\cdots$ & $\cdots$ & 61.4 & 0.96 & 0.94 \\
\hline \hline
\end{tabular}

estingly, no deviations similar to those reported in Refs. 8 and 13 have been observed between our experimental data and the ASD and (modified) $\delta \gamma$ scheme predictions.

In the present article, we extend the brief discussion of structural and short-time diffusion properties of chargestabilized suspensions of very monodisperse fluorinated latex spheres given in Ref. 4. In Ref. 4 experimental results had been shown only for a low-salt and high-salt sample at a single colloid concentration. In order to characterize in more detail the effect of the strength of the electrostatic repulsion on the structure and hydrodynamics of charge-stabilized colloidal suspensions, an experimental study that covers a broader range of colloid concentrations from highly ordered systems to dilute suspensions is required. Such an extended experimental study is included in the present paper where we discuss latex suspensions for various colloid concentrations, considered both in the low-salt and high-salt regimes. We have performed measurements of the form factor, static structure factor, diffusion function, hydrodynamic function, and of the dynamic scattering function using synchrotron radiation. We show that the experimental data obtained for $S(q), D(q)$, and $H(q)$ are in good agreement with our $\delta \gamma$ theory and ASD model calculations for all colloid and salt concentrations. We also discuss possible reasons for the discrepancy observed between our experimental data on $H(q)$, and the ones published previously in Refs. 8 and 13. Upper and lower boundaries for the peak height of $H(q)$ are discussed in dependence on colloid and salt concentrations.

The paper is organized as follows: Experimental details on the colloid system and on the experimental setup are given in Sec. II. Section III summarizes the theoretical background and explains the dynamic simulation method. Our experimental and theoretical results are compared and discussed in Sec. IV. Finally, our conclusions are presented in Sec. V.

\section{EXPERIMENT}

\section{A. Characterization of samples}

The fluorinated latex spheres were prepared according to a standard soap-free emulsion polymerization. ${ }^{26}$ The hydro- dynamic radius of the spheres measured by means of dynamic light scattering amounts to $80 \mathrm{~nm}$. The radius determined from the form factor measurement in dilute aqueous suspensions using SAXS and a homogeneous sphere model is $78.5 \mathrm{~nm}$, with a size polydispersity smaller than $2 \%$. In water, the latex spheres are negatively charged since protons are dissociated from the sulphate and sulfonate acid groups on their surfaces. The suspension was kept in contact with an ion exchange resin (Amberlite MB3 monobed resin, Sigma) for $10 \mathrm{~h}$ in order to decrease its ionic strength. After this treatment, the suspension has crystallized. From this stock solution, two samples of a total $\mathrm{NaCl}$ concentration of $0.5 \mathrm{mM}$ [low-salt (LS) series] and $50 \mathrm{mM}$ [high-salt (HS) series], respectively, were prepared by adding a small amount of concentrated $\mathrm{NaCl}$ solution. Both sample series are labeled in the present work as A, B, and C, in the order of decreasing colloid concentration. All important parameters of these samples are given in Table I. The ionic strength of the LS series was chosen such that even the most concentrated sample, i.e., LS-A, is in the fluid state. The samples of the LS series are within the volume fraction range where hydrodynamic screening had been reported to occur. ${ }^{8,13}$ In our XPCS experiments, the samples were filled into quartz capillaries of a diameter of $1.5 \mathrm{~mm}$ and sealed with glue.

\section{B. Experimental setup and methods}

The XPCS experiment was performed at the Troika III of the Troika beamline ID10A at the European Synchrotron Radiation Facility (ESRF) Grenoble. A schematic view of the setup and experimental details have been given elsewhere. ${ }^{27}$ The synchrotron was running in a $2 / 3$ filling mode with an average current of about $200 \mathrm{~mA}$. Measurements were performed at $7.990 \mathrm{keV}$, and at a wavelength of $\lambda=1.55 \AA$.

The coherent beam is provided by a $12 \mu \mathrm{m}$ pinhole placed at $25 \mathrm{~cm}$ distance from the sample. With this setup the primary beam intensity was about $10^{9}$ photons/s at $100 \mathrm{~mA}$. The sample was mounted in an evacuated smallangle scattering chamber and the scattered photons are guided through a vacuum flight path to the detector stage, 
resulting in a sample-to-detector distance of $3.33 \mathrm{~m}$. The $\mathrm{x}$-ray photons have been detected by a Bicron scintillation counter equipped with an adjustable pair of slits of 150 $\times 150 \mu \mathrm{m}^{2}$. Correlation functions were calculated in real time via an ALV-5000 digital correlator.

Our SAXS measurements have been performed using the same experimental setup, but for part of the runs a larger pinhole defining the incident beam has been used.

\section{THEORETICAL BACKGROUND}

\section{A. One-component model system}

In calculating static and dynamic properties of chargestabilized colloidal spheres, we need to model the interaction potential, and to specify the properties of the embedding solvent. All our calculations are based on the standard onecomponent macroion fluid (OMF) model. In this continuum model, the colloids are described as uniformly charged hard spheres, interacting by the effective Derjaguin-LandauVerwey-Overbeek (DLVO)-like pair potential ${ }^{28,29}$

$$
\frac{u(r)}{k_{B} T}=L_{B} Z^{2}\left(\frac{e^{\kappa a}}{1+\kappa a}\right)^{2} \frac{e^{-\kappa r}}{r}, \quad r>2 a .
$$

For the strongly charged polystyrene spheres studied in this work, $Z$ must be interpreted as an effective colloid charge number that accounts, to some extent, for nonlinear screening effects. Furthermore, $a$ is the hard-core radius of a colloidal sphere and $L_{B}=e^{2} /\left(\varepsilon k_{B} T\right)$ is the Bjerrum length for a suspending fluid of dielectric constant $\varepsilon$. For water at room temperature, $L_{B}=0.71 \mathrm{~nm}$. The solvent is thus described as a structureless continuum, characterized solely by $\varepsilon$ and the shear viscosity $\eta_{0}$. The latex suspensions in the present study are closed systems with no contact to an electrolyte reservoir (i.e., no Donnan equilibrium here). The electrostatic screening parameter $\kappa$ for a closed system is given by

$$
\kappa^{2}=\frac{4 \pi L_{B}\left(n|Z|+2 n_{s}\right)}{1-\phi}=\kappa_{\mathrm{ci}}^{2}+\kappa_{s}^{2} .
$$

Here, $n$ is the colloid number density, $n_{s}$ is the number density of added 1-1 electrolyte, and $\phi=(4 \pi / 3) n a^{3}$ is the colloid volume fraction. As noticed in Eq. (6), $\kappa^{2}$ consists of a contribution, $\kappa_{\mathrm{ci}}^{2}$, due to counterions that are assumed here to be monovalent, and another contribution, $\kappa_{s}^{2}$, arising from added 1-1 electrolyte (e.g., $\mathrm{NaCl}$ ).

The factor $1 /(1-\phi)$ corrects for the free volume accessible to the screening microions, owing to the presence of colloidal spheres. It is of relevance for very dense suspensions only. Equation (6) has been derived, e.g., from a Poisson-Boltzmann spherical cell model calculation where the electrostatic potential has been linearized around its mean value. ${ }^{30,31}$ We are not concerned here with the ongoing discussion on how the effective charge in Eq. (5) is related to the bare charge, in particular, when nondilute suspensions of non-crystalline-like structure are considered, as in the present work. We rather use $Z$ as an adjustable parameter, selected by matching the peak height of the calculated static structure factor to the experimental one.

\section{B. Static properties}

The static structure factor, $S(q)$, can be determined from SAXS experiments, and from knowledge of the colloid particle form factor. To calculate $S(q)$ based on the OMF pair potential, we have solved numerically the Ornstein-Zernike integral equation using the well-established Rogers-Young (RY) and rescaled mean-spherical approximation (RMSA) closure relations. ${ }^{32-34}$ The RY scheme is known for its excellent structure factor predictions within the OMF model. The RMSA results for $S(q)$ are in most cases nearly identical to the RY ones, provided a somewhat different value of $Z$ is used. For systems of strongly correlated particles $Z_{\mathrm{RMSA}}$ is typically larger than $Z_{\mathrm{RY}}$ but the opposite ordering may be found for sufficiently weak interparticle correlations. ${ }^{12}$ RMSA calculations of $S(q)$, in particular, are computationally very fast. Therefore, the RMSA is still in use as a convenient tool when extensive structure factor scans are required over a wide range of interaction parameters.

\section{Theory and computer simulation of dynamic properties}

The dynamic structure factor of spherical particles, $S(q, t)$, can be determined using XPCS. It is defined by the equilibrium ensemble average

$$
S(q, t)=\left\langle\frac{1}{N} \sum_{j, k=1}^{N} \exp \left\{i \mathbf{q} \cdot\left[\mathbf{R}_{j}(0)-\mathbf{R}_{k}(t)\right]\right\}\right\rangle .
$$

Here, $\boldsymbol{q}$ is the scattering vector, $N$ is the number of colloids in the scattering volume, and $\boldsymbol{R}_{j}(t)$ is a vector pointing to the center of the $j$ th colloidal sphere taken at time $t$.

At short correlation times ${ }^{1,2,28}$

$$
\frac{S(q, t)}{S(q)}=\exp \left[-q^{2} D(q) t\right],
$$

where $D(q)$ is the short-time diffusion function defined in Eq. (2). The hydrodynamic function can be inferred, using Eq. (3), from a short-time XPCS experiment combined with a SAXS measurement of $S(q)=S(q, t=0)$.

The volume fractions of most of our samples are so large that in theoretical calculations of $H(q)$ and $S(q, t)$ one needs to account for three-body and higher-order HI contributions. To compute $H(q)$ on the basis of the OMF model, we have used the zeroth-order version of the $\delta \gamma$ expansion theory developed by Beenakker and Mazur. ${ }^{19}$ As noted in the Introduction, this method requires the static structure factor as input. The latter is calculated in our study using the RY and RMSA integral equation schemes. In $\delta \gamma$ theory the actual charged sphere $S(q)$ enters only into the wave-numberdependent distinct part, $H_{d}(q)$, of $H(q)$. The constant selfpart, $D_{s} / D_{0}$, in Eq. (1) is approximated more roughly using the value for neutral hard spheres at the same volume fraction independent of the actual values of the sphere charge and the screening parameter. ${ }^{19}$ Yet, through an extensive comparison between simulation, experiments, and $\delta \gamma$ theory predictions, we have found that the relative amplitudes and the phases of the wave-number undulations in $H(q)$, as embodied in $H_{d}(q)$, are quite well captured by the $\delta \gamma$ theory. ${ }^{4,12}$ 
This finding allows us to improve the $\delta \gamma$ scheme through substituting the $\delta \gamma-D_{s}$ by an accurate simulation prediction. This substitution procedure is computationally less expensive than a full simulation of $H(q)$. Application of the hybrid $\delta \gamma$-ASD scheme amounts practically to an upward shift of the bare $\delta \gamma-H(q)$ by a small to moderately large amount, owing to the fact that the charged-sphere short-time selfdiffusion coefficient is larger than the hard-sphere value for the same density. ${ }^{2,4,7}$ Even when the $\delta \gamma$ scheme is used without any simulation correction for $D_{s}$, it is usually sufficient to predict, on a semiquantitative level, general trends in the behavior of $H(q)$ and $D(q)$.

Our simulation calculations of $D(q), H(q)$, and of $S(q, t)$ for charge-stabilized spheres interacting by the OMF pair potential are based on an accelerated ASD simulation code for Brownian spheres developed by Banchio and Brady. ${ }^{25}$ This code enables us to simulate a larger number of Brownian spheres, typically up to 1000 , which leads to an improved statistics. The essentials of this simulation method are explained in Ref. 25. To speed up the computations of $H(q)$, which as a short-time property requires for its computation to perform a static equilibrium average only, we have generated a set of equilibrium configurations of spheres using a Monte Carlo simulation code for charged spheres, and a molecular dynamics simulation code for neutral hard spheres. ${ }^{35}$ The many-body HIs have been computed using the ASD scheme. To correct for finite-size effects arising from periodic boundary conditions, ASD simulations of $H(q)$ and $S(q, t)$ have been repeated for each system using an ascending number, $N=125,343$, and 860 , of spheres in the basic simulation box. From this sequence of simulations, an extrapolation to the thermodynamic limiting form of $H(q)$ is made on employing the finite-size scaling formula,

$$
H(q)=H_{N}(q)+1.76 S(q) \frac{\eta_{0}}{\eta_{\infty}(\phi)}(\phi / N)^{1 / 3} .
$$

This expression was initially proposed for hard spheres by Ladd et $a l .{ }^{36}$ The finite-size scaling form used to obtain the $H(q)$ of a macroscopic system requires thus, in addition to $H_{N}(q)$, to compute the high-frequency-limiting shear viscosity, $\eta_{\infty}$, of a weakly sheared suspension in the limit of zero Peclet number. The simulation data obtained for the various $N$ collapse neatly on a single master curve once Eq. (9) has been used. The resulting master curve is identified with the finite-size corrected form of $H(q)$.

\section{RESULTS AND DISCUSSION}

As noted in the Introduction, the purpose of this article is to explore the interplay of electrosteric and hydrodynamic forces in their influence on the short-time translational diffusion properties of charge-stabilized colloidal spheres. In the following we discuss our experimental findings for the LS and HS sample series, and compare them to ASD simulation results and theoretical work. The simulation and theory results have been obtained using the one-component macrofluid model described in Sec. III A, for the system parameters summarized in Table I. We first discuss static properties

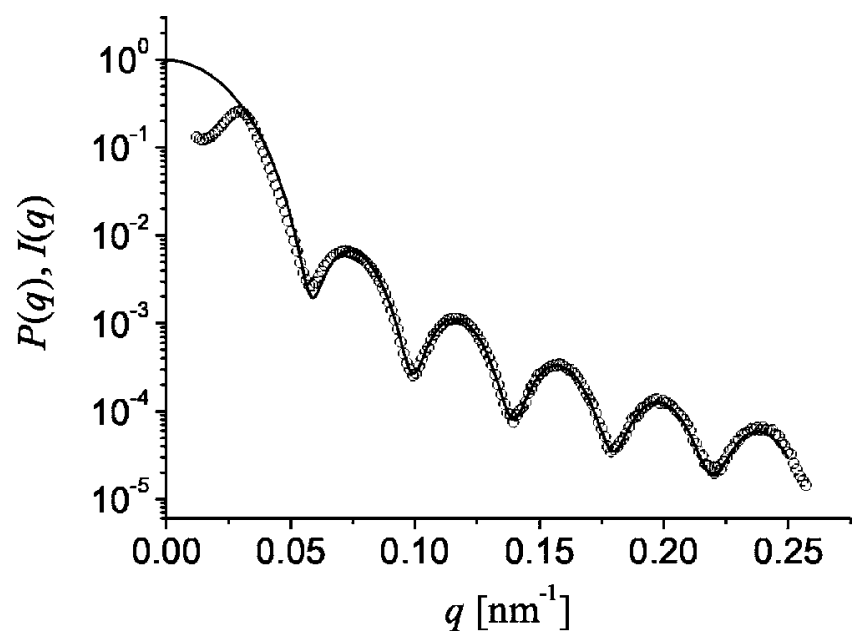

FIG. 1. Scaled mean measured intensity, $I(q)$, of sample LS-A (symbols) obtained using SAXS. The solid line represents the size-averaged form factor, $P(q)$, for a Gaussian-distributed collection of homogeneous spheres with a mean radius of $78.5 \mathrm{~nm}$ and a relative standard deviation in the size distribution of $2 \%$.

since these are basic to the dynamic calculations. In the second part of this section short-time dynamic properties are studied.

\section{A. Form and static structure factors}

Figure 1 shows SAXS data of the scaled mean scattered photon intensity $I(q)=P(q) S(q)$, obtained for the most concentrated sample LS-A. These data reduce to the bare form factor, $P(q)$, at wave numbers $q>0.08 \mathrm{~nm}^{-1}$. We have used a concentrated sample to gain an improved signal-to-noise ratio. The well-pronounced multiple oscillations in $I(q)$ at larger $q$ are indicative of a low-polydispersity sample. The solid line is a form factor fit assuming homogeneous spheres of Gaussian size distribution. As can be seen, a good fit of the SAXS data is achieved for a mean static radius of $a$ $=78.5 \mathrm{~nm}$, and a relative standard deviation in the size distribution of $2 \%$. The deviations of the SAXS $I(q)$ from the model $P(q)$ at small $q$ are due to non-negligible electrosteric particle interactions that give rise to low- $q$ values of $S(q)$ smaller than 1 . However, in all theory and computer simulation calculations discussed in this paper, a common hardsphere radius $a=82.5 \mathrm{~nm}$ has been used (cf. Table I). This value can be regarded as an effective hard-sphere radius, and it allows for a consistent description of all our static and dynamic experimental data. We argue that a possible reason for this difference in the particle radius is due to the fact that our polymeric colloidal particles might not be perfect homogeneous hard spheres, but rather hairy spheres with charged polymer chains sticking out of the collapsed polymer core, which has a higher electron density difference. Therefore we also tried to fit the SAXS data for $I(q)$ with a form factor model describing core-shell particles with core radius of $78.0 \mathrm{~nm}$ and outer radius of $84.1 \mathrm{~nm}$, with a polydispersity in the core radius of $2 \%$, and an inner-to-outer shell scattering strength ratio of 27.4. This fit describes the $I(q)$ curves equally well as the fit for homogeneous hard sphere shown in Fig. 1. Thus, the radius of $82.5 \mathrm{~nm}$ used in the analysis can 


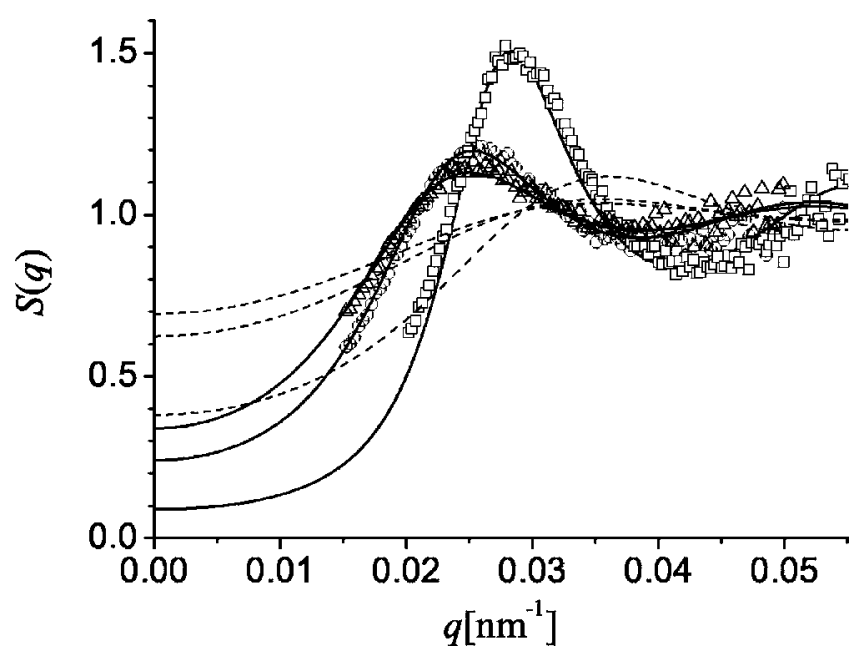

FIG. 2. SAXS static structure factor, $S(q)$, of low-salt samples LS-A ( $\square)$, LS-B $(\bigcirc)$, and LS-C $(\triangle)$. The colloid concentration decreases from LS-A to LS-C (cf. Table I). The concentration of added $\mathrm{NaCl}$ is $0.5 \mathrm{mM}$. The solid lines are the corresponding RY fits. The dashed lines are PY calculations of $S(q)$, for neutral hard spheres at the same volume fractions as in the corresponding LS cases.

be considered as an effective hard-sphere radius of our coreshell particles.

Experimental and theoretical results for the static structure factor, $S(q)=I(q) / P(q)$, of the low-salt and high-salt samples are depicted in Figs. 2 and 3, respectively.

Our SAXS data for the low-salt series of samples LS-A, LS-B, and LS-C (with decreasing colloid concentration from LS-A to LS-C) are well captured by the RY structure factors (solid lines), using values of the effective charge $Z$ as listed in Table I. Note that the effective particle charge is essentially the only adjustable parameter. It is obtained from matching the height of the principal peak of the RY-S $(q)$ to the experimental one. In Figs. 2 and 3 only the RY-calculated $S(q)$ have been included, since these are practically coincident with the ASD simulation data on $S(q)$, for exactly the same system parameters. In addition, the RMSA-calculated

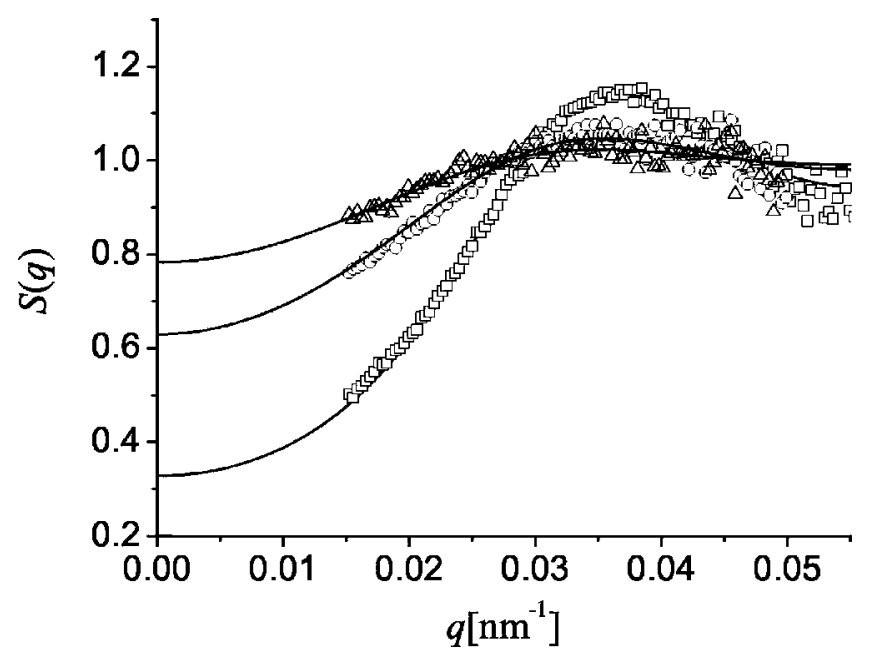

FIG. 3. SAXS static structure factor of high-salt colloidal suspensions HS-A $(\square)$, HS-B $(\bigcirc)$, and HS-C $(\triangle)$ for $50 \mathrm{mM}$ of added $\mathrm{NaCl}$. The solid lines are the corresponding RMSA/RY fits. The PY structure factor curves calculated for genuine hard spheres of the same volume fractions are practically coincident with the solid lines. structure factors become nearly identical to the RY ones, provided somewhat different values of $Z$ are used. These RMSA-fit values for $Z$ are included in Table I.

The structure factors of the LS samples are typical for charge-stabilized suspensions: the peak height, $S\left(q_{m}\right)$, at wave number $q_{m}$ increases with increasing concentration. The peak location $q_{m}$ moves out to larger values of $q$ when the particle concentration is increased, owing to the reduced mean interparticle distance. Likewise, the value of $S(q)$ at small $q$ becomes smaller at higher volume fractions owing to a smaller osmotic compressibility. It is interesting to compare the structure factors of charge-stabilized spheres to those of neutral hard spheres of the same size and volume fraction (see Fig. 2). The corresponding hard-sphere $S(q)$ has a significantly flatter $q$ dependence, since the structuring influence of the electrostatic repulsion is missing, and the peak position $q_{m}$ is located at a larger value of $q$. In the range of volume fractions and wave numbers spanned by the LS and HS samples, the Percus-Yevick (PY) hard-sphere structure factors depicted in Fig. 2 are practically coincident with the corresponding RY and ASD results for neutral hard spheres.

The electrostatic screening length, $\kappa^{-1}$, of the high-salt samples HS-A-HS-C is so small as compared to the sphere radius (cf. Table I) that their static structure factors are practically equal to those of neutral spheres. Therefore, hardly any changes in $S(q)$ are observed when $Z$ is varied over a broad range of values. The static structure factor fits of the HS series are, within the experimental noise level, of similar good quality as those of the LS samples. The high-salt samples are less ordered than their LS counterparts, as notified, e.g., by a shallower and broader maximum in $S(q)$.

The integral equation results for $S(q)$ displayed in Figs. 2 and 3 are used as input to the $\delta \gamma$ theory calculations of $H(q)$. Our results for $H(q)$ will be discussed in Secs. IV C-IV E.

\section{B. XPCS dynamic scattering function evaluation}

In Fig. 4, the XPCS normalized electric field autocorrelation function, $g^{(1)}(q, t)$, of the low-salt sample LS-A is plotted as a function of reduced time $D_{0} q^{2} t$. Three wave numbers located below, at, and above the peak position, $q_{m}$, of $S(q)$, respectively, are considered. The following features can be noticed: The decay of the autocorrelation function at $q_{m}$ is the slowest one, consistent with the minimum of $D(q)$ at the peak position of $S(q)$. Furthermore, the decay of $g^{(1)}\left(q_{m}, t\right)$ appears to be single exponential over the whole time window displayed in Fig. 4. In contrast to this observation, deviations from a single-exponential decay are clearly observed at longer times for wave numbers located below and above $q_{m}$. The nonexponential decay at longer times can be attributed to the influence of electrosteric and hydrodynamic interaction forces acting between neighboring particles that slow down the collective diffusion process relative to the fast short-time decay. The linear short-time window for $\ln g^{(1)}(q, t)$ as a function of correlation time is broad enough at all three wave numbers so that $D(q)$ can be determined to good accuracy. The electric field autocorrelation functions shown in Fig. 4 have been obtained from the measurements 


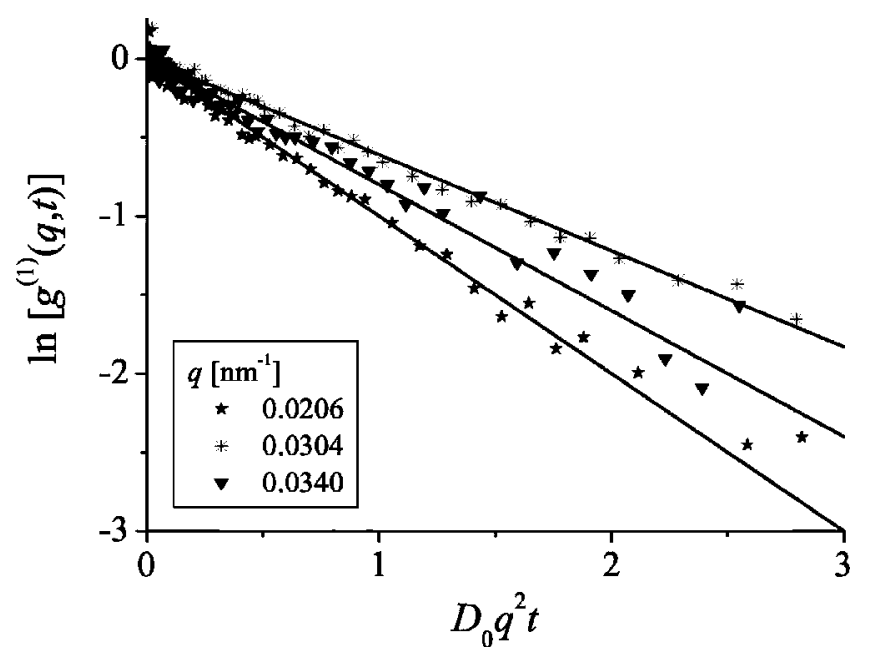

FIG. 4. Semilogarithmic plot of the XPCS normalized electric field autocorrelation function of low-salt sample A vs reduced time for three values of the scattering wave number as indicated. The solid lines are fits to the short-time initial slope of $\ln g^{(1)}(q, t)$. From this slope the short-time diffusion function $D(q)$ is inferred.

of the x-ray intensity time autocorrelation function using the standard Siegert relation of Gaussian-distributed random variables.

\section{Hydrodynamic function: Simulation and theory}

Before we start to discuss our experimental data for $D(q)$ and $H(q)$, we first compare the $\delta \gamma$ theory predictions for the hydrodynamic function of low-salt samples $\mathrm{A}, \mathrm{B}$, and $\mathrm{C}$ to the corresponding ASD simulation results. Such a comparison is made in Fig. 5. We recall that the $\delta \gamma H(q)$ shown in this figure have been calculated using the RY static structure factors in Fig. 3. While the wave-number variations in the simulated $H(q)$ are well captured by the $\delta \gamma$ theory, the ASD $H(q)$ is overall underestimated. As it was explained in Sec. III B this deviation occurs since the self-part, $D_{s}$, of the $H(q)$

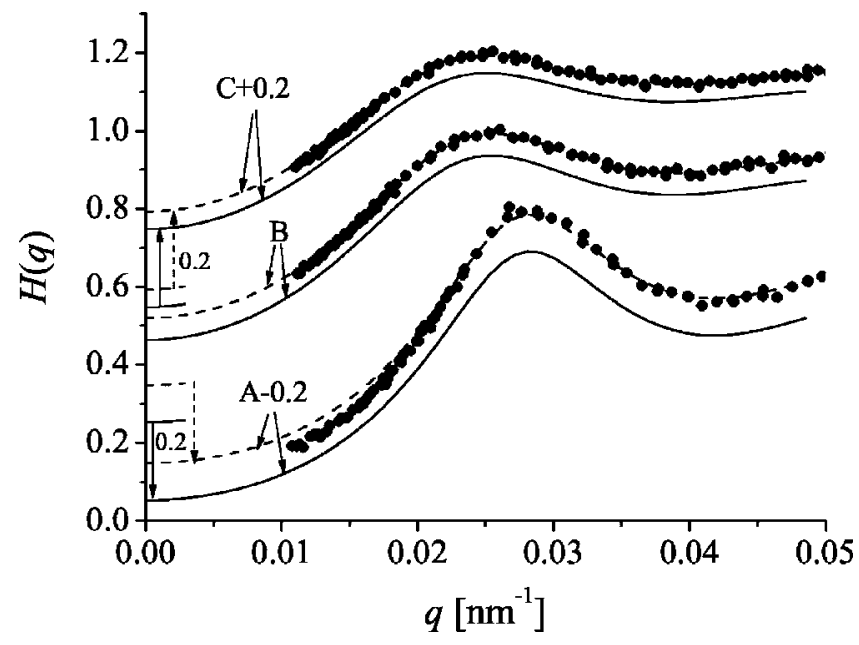

FIG. 5. Hydrodynamic function $H(q)$ of low-salt samples LS-A, LS-B, and LS-C. Comparison between finite-size corrected ASD simulation data (black filled circles), $\delta \gamma$ theory results with RY input for $S(q)$ (solid lines), and hybrid $\delta \gamma$ theory calculations (dashed lines) where the self-part, $D_{s}$ of $H(q)$ is computed using ASD. The curves of samples LS-A and LS-C are vertically shifted relative to those of sample LS-B by \pm 0.2 to improve visibility.

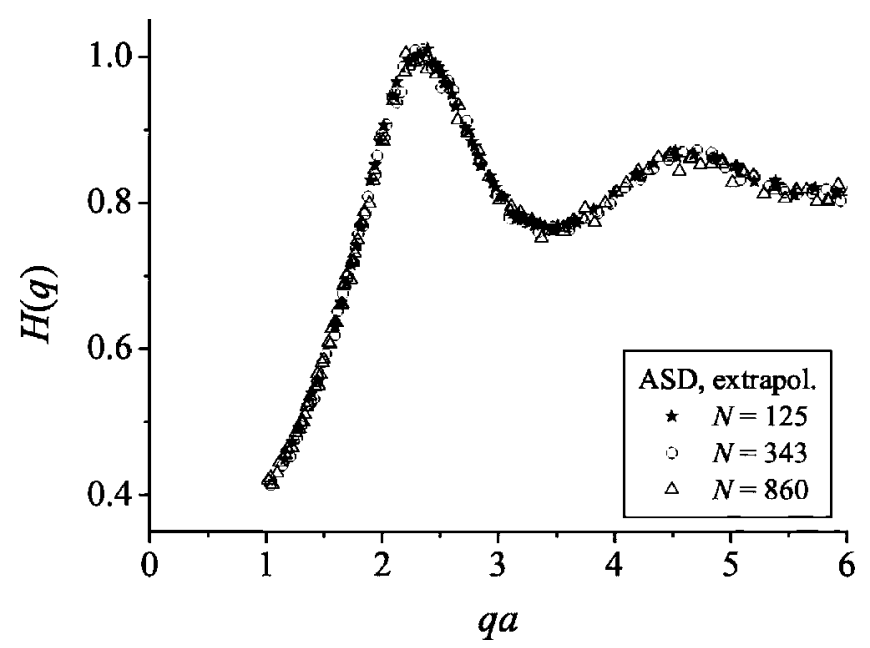

FIG. 6. Finite-size corrected ASD simulation results for the hydrodynamic function of the low-salt system LS-A, obtained for numbers, $N$, of spheres in the basic simulation box as indicated. On using the scaling form in Eq. (9), all simulation data neatly collapse on a master curve.

of charged spheres is approximated in the $\delta \gamma$ theory by the value for hard spheres at the same concentration, irrespective of particle charge and ionic strength. The RY structure factor of charge-stabilized particles enters in the $\delta \gamma$ theory only through the distinct part, $H_{d}(q)$, of $H(q)$. The short-time selfdiffusion coefficient of neutral hard spheres depends only on $\phi$. Up to $\phi \approx 0.3$ it is well described by the second-order virial form $D_{S} / D_{0}=1-1.832 \phi-0.219 \phi^{2}{ }^{37}$ For volume fractions larger than 0.3 , this second-order expression becomes less accurate. ${ }^{34}$ The accuracy of the $\delta \gamma$ theory is improved when it is combined with more accurate ASD simulation data for the self-part, $D_{s}$ (cf. Table I) of charged spheres. Since $D_{s}$ is larger for charged particles than for neutral ones, ${ }^{2,7}$ this correction amounts to an upward shift of the $\delta \gamma H(q)$ by an amount which is larger for higher particle concentration. The good overall agreement achieved by this hybrid $\delta \gamma$ theory method with the more elaborate ASD simulations is nicely illustrated in Fig. 5.

The ASD- $H(q)$ included in Fig. 5 have been obtained using the finite-size correction formula given in Eq. (9). This system-size scaling procedure has been initially used for hard spheres only. We have verified that this procedure works well also in the case of charged particles, by considering simulation runs made using an increasing number $N=125-860$ of particles per basic simulation box. For sample LS-A, this finite-size scaling prescription is illustrated in Fig. 6. As can be seen the scaling procedure leads to a $H(q)$ practically independent of $N$.

\section{Short-time diffusion: Comparison with experiment}

In the following we discuss our experimental results for $D(q)$ and $H(q)$ in comparison to simulation and theory. The XPCS data for $D(q)$ have been determined, on employing Eq. (2), using a standard cumulant analysis of $g^{(1)}(q, t)$ and an extrapolation to the short-time limit. The electric field autocorrelation function, in turn, was obtained from the measured intensity autocorrelation function using the Siegert relation. The values for $D(q)$ deduced from such an analysis 


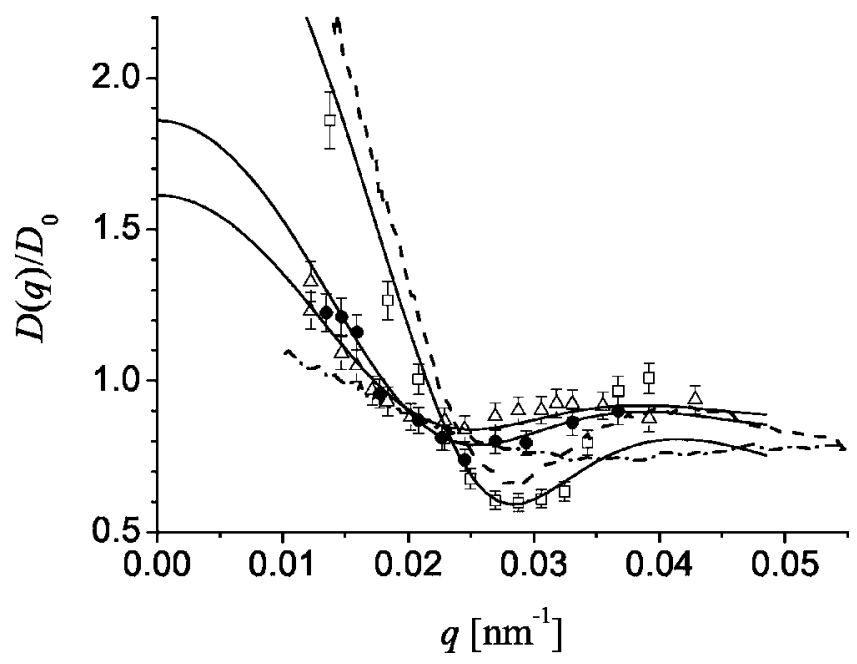

FIG. 7. XPCS data for the dimensionless diffusion function, $D(q) / D_{0}$, vs wave number $q$ for the low-salt samples LS-A $(\square)$, LS-B $(\bullet)$, and LS-C $(\triangle)$. The $\mathrm{NaCl}$ concentration is fixed to $0.5 \mathrm{mM}$. The solid lines are the $\delta \gamma$ theory predictions with RY input for $S(q)$. The dashed line is the ASD prediction for LS-A. The dashed-dotted curve is the ASD prediction for neutral hard spheres of the same concentration as sample LS-A (all volume fractions are listed in Table I).

depend for low-salt systems sensitively on the employed time window. Selecting a too large time window may lead to values for $D(q)$ that are smaller than the true short-time values, since contributions to the decay of the dynamic structure factor, $S(q, t)=g^{(1)}(q, t) / g^{(1)}(q, 0)$, can be picked up which describe sublinear diffusion that occurs at longer times. In our analysis of the scattering data, we have accumulated sufficient statistics to determine $D(q)$ to good precision from the genuine first cumulant of the dynamic scattering function. All systems considered in this work are in a homogeneous and isotropic fluid state, with no indications whatsoever in the dynamic scattering function for an onset of crystallization, or for nonergodic dynamic arrest that occurs in gels or glasses. The structure factor peak heights of all samples are smaller than two (cf. Figs. 2 and 3).

The XPCS data for the dimensionless diffusion function, $D(q) / D_{0}$, of the low-salt and high-salt samples A, B, and C are shown in Figs. 7 and 8, respectively. The free-particle diffusion coefficient $D_{0}$ has been determined using dynamic light scattering performed on very dilute colloidal suspensions with a large amount of salt added to screen out electrostatic interactions. In this way the value for $D_{0}$ has been determined to $2.69 \times 10^{-8} \mathrm{~cm}^{2} / \mathrm{s}$. The experimental data (symbols) are compared in these figures to $\delta \gamma$ theory predictions (solid lines), and to ASD simulations (dashed lines). ASD results are shown in Figs. 7 and 8 for the most concentrated samples LS-A and HS-A only. Moreover, hybrid- $\delta \gamma$ results for $H(q)$ are not included since these are nearly coincident with the ASD data (cf. Fig. 5). Within the experimental errors both the $\delta \gamma$ theory and ASD simulations results for $D(q)$ are in good accord with the experimental data.

Experimental, simulation, and $\delta \gamma$ theory results for the hydrodynamic function, $H(q)$, of the low-salt and high-salt series are shown in Figs. 9 and 10. The experimental data for $H(q)$ have been determined by multiplying the XPCS $D(q)$, in accord with Eq. (3), by the integral equation static struc-

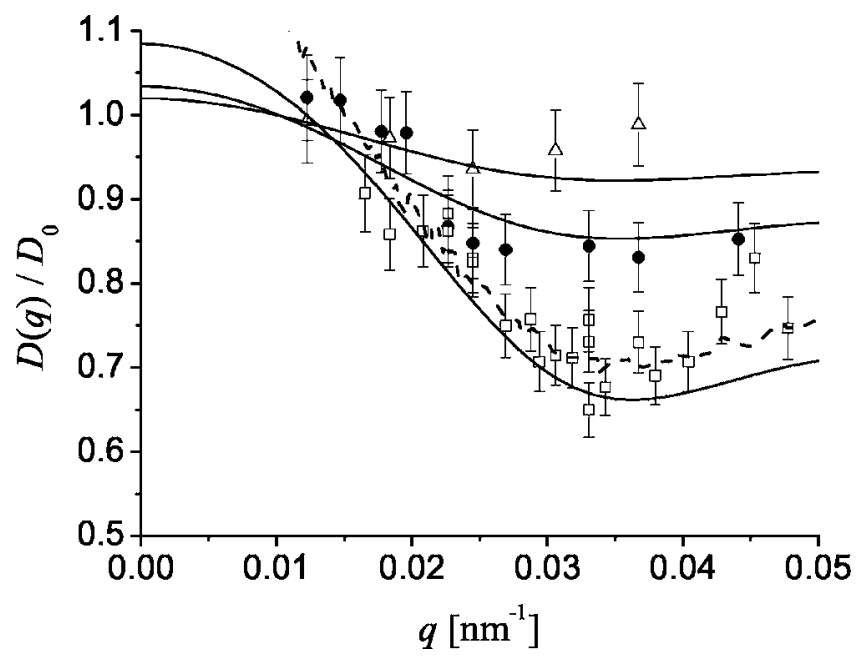

FIG. 8. Same as in Fig. 7 but for the high-salt samples HS-A ( $\square$ ), HS-B $(\bullet)$, and $\mathrm{HS}-\mathrm{C}(\triangle)$, with $\mathrm{NaCl}$ concentration fixed to $50 \mathrm{mM}$ (see Table I). The diffusion functions of the HS-series systems are practically equal to those of neutral hard spheres at the same volume fractions. Dashed line: ASD prediction for the most concentrated sample HS-A.

ture factors shown in Figs. 2 and 3. It is evident from Figs. 7 and 9 that the ASD predictions for the $D(q)$ and $H(q)$ of hard spheres are clearly different from those of the low-salt series. In particular, the maximum in $H(q)$ of the low-salt samples is
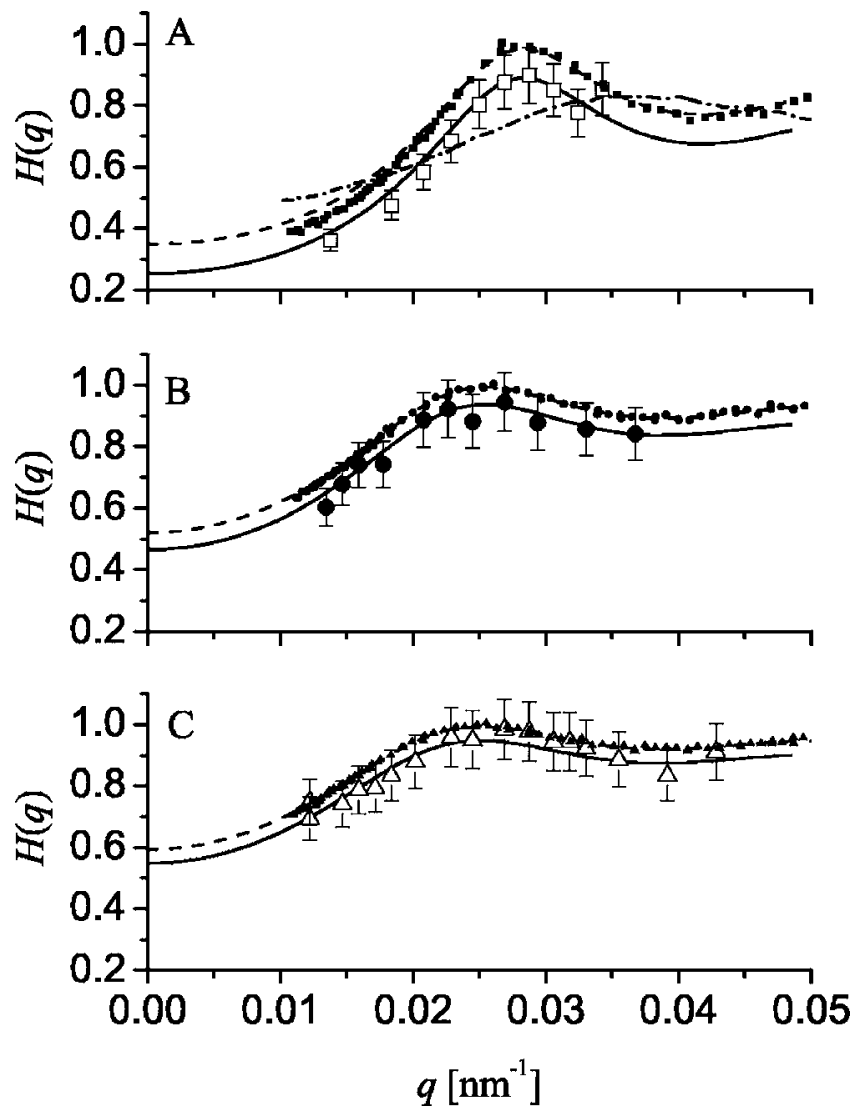

FIG. 9. Large symbols: experimentally determined hydrodynamic function $H(q)$ of low-salt samples LS-A $(\square)$, LS-B $(\bullet)$, and LS-C $(\triangle)$. The meaning of the symbols and the system parameters are the same as in Fig. 7. (-) lines: $\delta \gamma$ theory results with RY input. $(\bigcirc \circ, \triangle \triangle \triangle)$ : ASD simulations. (---): hybrid $\mathrm{ASD}-\delta \gamma$. (--.): ASD result for hard spheres at the volume fraction of sample LS-A. 


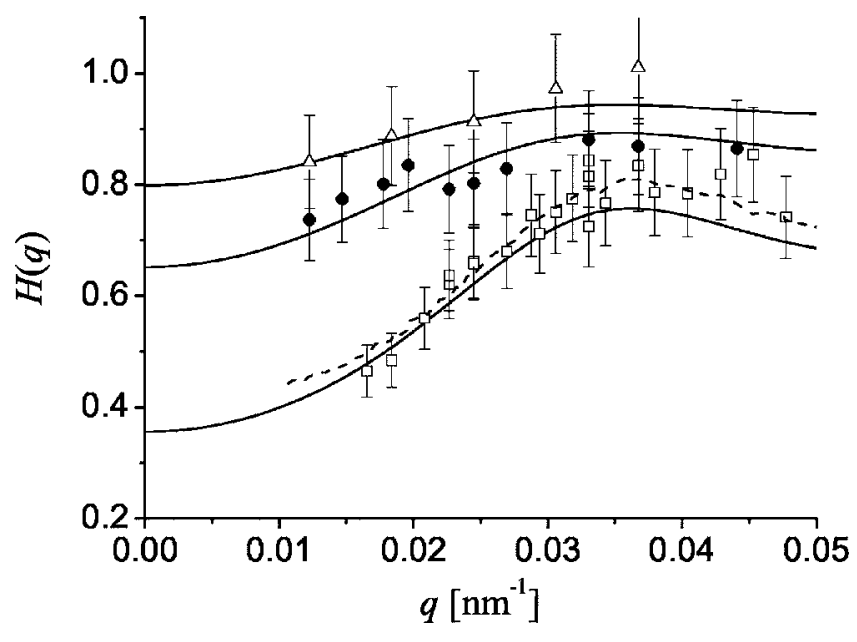

FIG. 10. Hydrodynamic function of high-salt samples HS-A ( $\square)$, HS-B (๑), and HS-C $(\triangle)$. The symbols and system parameters are the same as in Fig. 8. (-): $\delta \gamma$ theory predictions. (---): ASD simulation result for the most concentrated sample HS-A.

consistently larger than that of equal-concentration hardsphere suspensions and the peak position is located at a smaller wave number. This observation reflects the smaller likelihood of finding two or more charged particles at close distances. It should be noticed that the low-salt samples considered here have volume fractions in the range where hydrodynamic screening had been postulated to occur. ${ }^{8,13}$ Contrary to very dilute suspensions of strongly charged particles at low salinities where the peak height, $H\left(q_{m}\right)$, of $H(q)$ is larger than 1 and increases with concentration, the $H\left(q_{m}\right)$ of the low-salt series at higher concentrations is smaller than 1 . Moreover, the peak height decreases with increasing volume fraction. Indeed, ASD simulations of very dense chargestabilized suspensions predict values of $H\left(q_{m}\right)$ that are smaller than 1 , and decrease with increasing concentration similar to hard spheres. ${ }^{12}$ But even in the case of dense charge-stabilized systems $H\left(q_{m}\right)$ is consistently larger than the peak height of neutral spheres, and the peak position is found at smaller wave numbers. This generic behavior is observed in all our simulation and theory calculations.

As we have noted before, the electrostatic screening length of the high-salt samples is small enough $\left(\kappa^{-1}\right.$ $<0.01 a$ ) that $D(q)$ and $H(q)$ are practically equal to the equal-density hard-sphere functions. In fact, the peak height of the experimental $H(q)$ of the high-salt series agrees with the linear form given in Eq. (4).

\section{E. Boundaries on the peak height of $H(q)$}

We proceed now with a general discussion of the density and ionic strength dependence of $H\left(q_{m}\right)$ for charge-stabilized spheres interacting by the screened Coulomb potential defined in Eq. (5). Two limiting cases are particularly interesting: neutral hard spheres (infinite salt limit), and de-ionized charge-stabilized systems with all excess ions removed. Results for $H\left(q_{m}\right)$ vs $\phi$ that are representative for chargestabilized suspensions and suspensions of hard spheres are displayed in Fig. 11.

The solid line describes the linear density dependence of

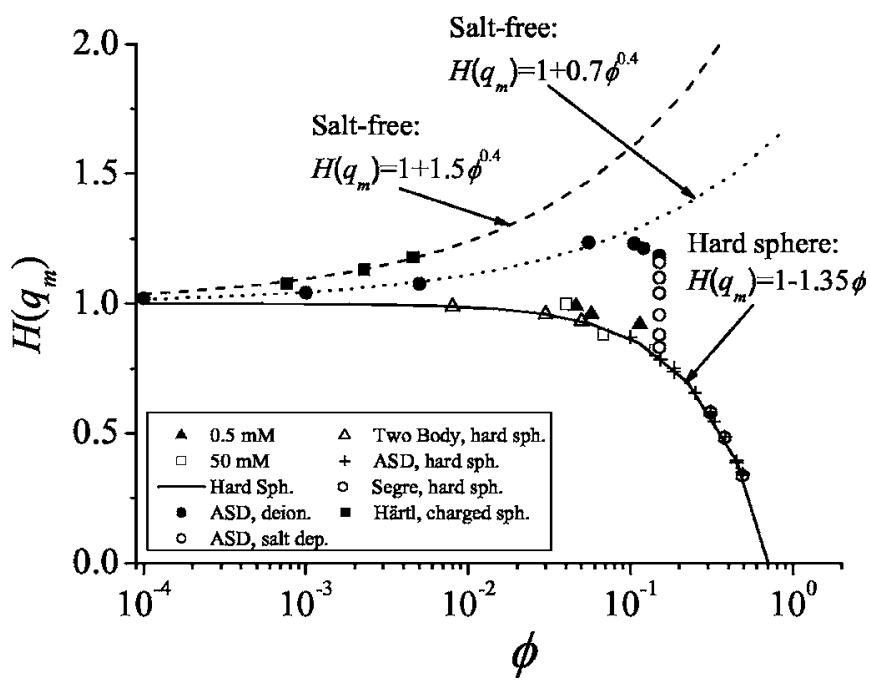

FIG. 11. Maximum of $H(q)$ as a function of colloid volume fraction $\phi$. (---): de-ionized suspensions [with experimental data of Härtl et al. (Ref. 17) (ם) overlayed]. (-): hard spheres. Further included are our experimental data for $0.5 \mathrm{mM}(\boldsymbol{\Delta})$ and $50 \mathrm{mM}(\square)$ of added $\mathrm{NaCl}$. ( $)$ : ASD data for a salt-free system; $(\mathrm{O})$ : ASD data for a system at fixed colloid concentration with 1-1 electrolyte added (the amount increases from top to bottom). System parameters used in the simulations: Bjerrum length $L_{B}=5.62 \mathrm{~nm}$, sphere radius $a=100 \mathrm{~nm}$, and effective charge $Z=100$ (assumed fixed). Additionally displayed are dynamic light scattering data of Segre et al. (Ref. 38) (O), lowdensity calculation results with full two-body HIs included (Ref. 18) $(\triangle$ ), and ASD results (Ref. 35) (+) for colloidal hard spheres.

the hard-sphere $H\left(q_{m}\right)$ according to Eq. (4), plotted on a semilogarithmic scale. Results for $H\left(q_{m}\right)$ of colloidal hard spheres obtained from ASD simulations, ${ }^{35}$ dynamic and static light scattering (Segre et al. ${ }^{38}$ ), and from low-density calculations with full two-body HIs included ${ }^{18}$ are seen to be in perfect agreement with this linear law. In addition, the experimental values for $H\left(q_{m}\right)$ of the high-salt samples HS-A, B, and C are practically located on the hard-sphere curve. The peak values of the low-salt samples are only a little bit larger than the corresponding hard-sphere values owing to the fact that significant electrostatic screening occurs already for $0.5 \mathrm{mM}$ of added salt. However, we recall that the location of the LS-series peaks of $H(q)$ and $S(q)$ are strongly different from those of hard spheres. According to Fig. 11, the values of $H\left(q_{m}\right)$ for charged spheres are bounded from below by the hard-sphere curve. An upper boundary is provided by the peak values of totally de-ionized systems. In this limiting case, however no unique boundary curve that applies simultaneously to all de-ionized suspensions exist. For dilute de-ionized systems where two-body HIs prevails, it was found from numerical calculations ${ }^{18}$ that $H\left(q_{m}\right)$ grows sublinearly in concentration according to ${ }^{18,34}$

$$
H\left(q_{m}\right)=1+p \phi^{0.4} \text {. }
$$

The coefficient $p>0$ is moderately dependent on $Z$ and $\kappa a$. The exponent 0.4 , however, is independent of these interaction parameters as long as the physical hard core of the spheres remains totally masked by the electrostatic repulsion. One can attribute the value $0.4 \approx 1 / 3$ for the exponent to the fact that in dilute de-ionized systems the location, $r_{m}$ $=2 \pi / q_{m}$, of the next-neighbor peak of the pair distribution function scales like $n^{-1 / 3}$, where $n$ is the number density of 

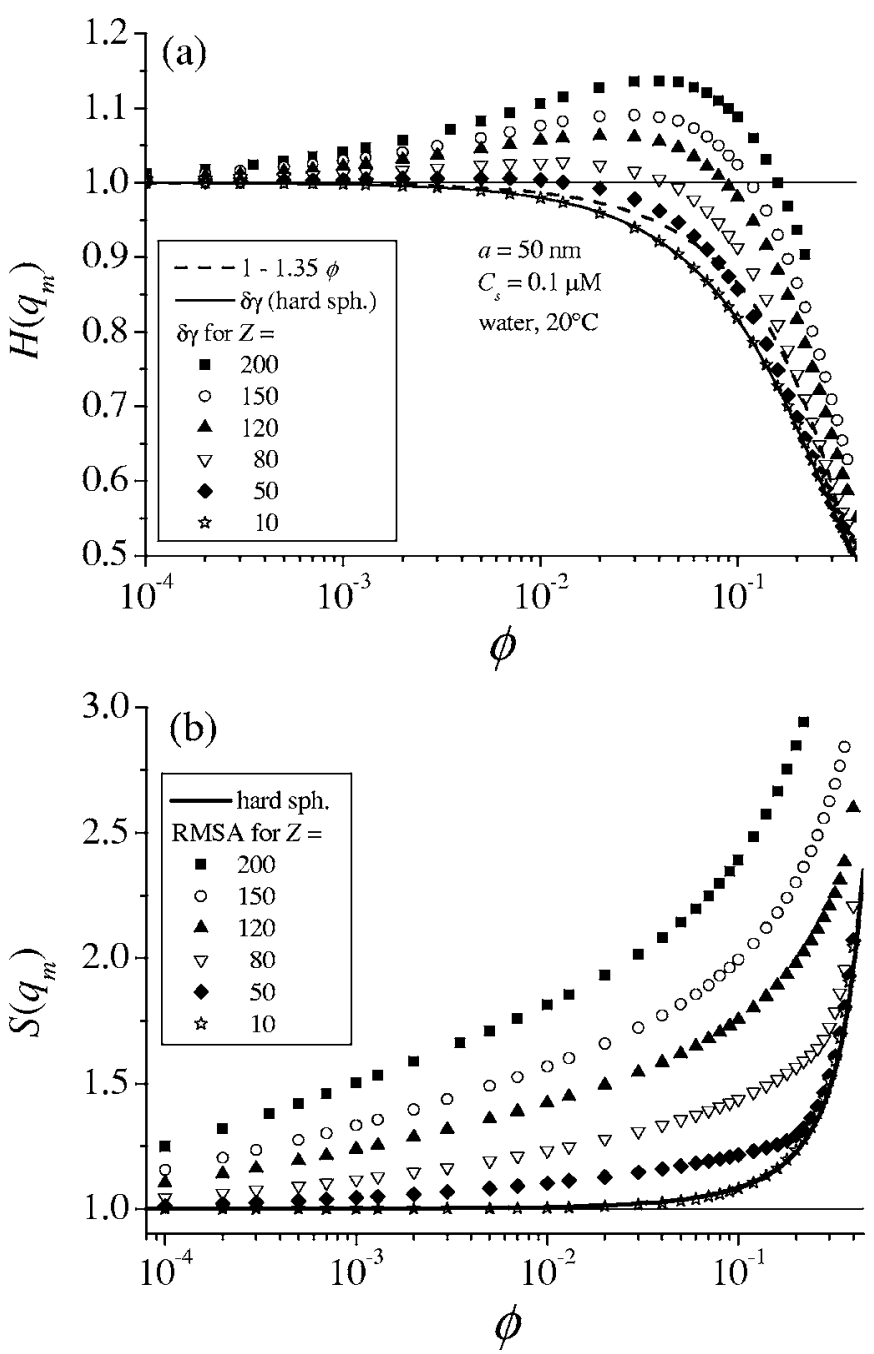

FIG. 12. (a) Concentration dependence of $H\left(q_{m}\right)$ for an aqueous chargestabilized suspension of effective particle charges as indicated. The results shown have been obtained using $\delta \gamma$ theory with RMSA input. Dashed line: hard-sphere Eq. (4). Solid line: $\delta \gamma$ theory prediction for hard spheres (see text). (b) Corresponding peak height, $S\left(q_{m}\right)$, of the RMSA static structure factor. The system parameters are $a=50 \mathrm{~nm}$ and $C_{s}=10^{-7} M$ (selfdissociation of water).

particles. ${ }^{18}$ The parameter $p$, on the other hand, depends on the details of the shape of the radial particle distribution function which is the reason for its nonuniversal behavior. For more strongly structured suspensions characterized by larger peak heights in $S(q)$, larger values of $p$ are observed. In particular, $p$ increases with increasing $Z$ for fixed particle radius [see Fig. 12(a)]. Figure 11 shows the sublinear lowdensity form in Eq. (10) for $p=1.5$ and 0.7 , respectively. We have assumed here that the effective particle charge is independent of concentration. The upper curve describes quantitatively the peak height of low-density aqueous suspensions of highly charged colloidal spheres that have been investigated experimentally by Härtl et al. ${ }^{17}$ The lower curve, with $p=0.7$, describes charged spheres of radius $a=100 \mathrm{~nm}$ and effective charge number $Z=100$, immersed in a lowdielectric solvent of Bjerrum length $5.6 \mathrm{~nm}$. This value for the effective charge was selected to describe a system that remains in the liquid state, even when de-ionized, up to a volume fraction of 0.15 . As seen, the ASD simulations of
$H\left(q_{m}\right)$ for this system are in agreement with the low-density form in Eq. (10) until three-body and higher-order hydrodynamic interaction effects come into play at larger concentration, which cause an enlarged hydrodynamic hindrance in the relative motion of neighboring spheres. As a consequence, $H\left(q_{m}\right)$ decreases then when $\phi$ is further increased. When electrolyte is added at fixed concentration (here, at $\phi=0.15$ ), $H\left(q_{m}\right)$ decreases monotonically towards the limiting hardsphere curve (see open circles in Fig. 11). Both our simulations and $\delta \gamma$ theory predict that the values of $H\left(q_{m}\right)$ for any system with added salt are located in between the zero-salt and hard-sphere curves.

At this place, it is appropriate to summarize the general behavior of the $H(q)$ of fluid charge-stabilized systems, namely, that values for $H\left(q_{m}\right)$ smaller than 1 which may increase or decrease with concentration can be attributed to (many-body) HIs effects without any need to postulate hydrodynamic screening. From our calculations and experiments, two generic ordering relations between chargedsphere (CS) systems and hard-sphere (HdS) systems are shown to be valid, namely,

$$
H^{\mathrm{CS}}\left(q_{m}, \phi\right)>H^{\mathrm{HdS}}\left(q_{m}, \phi\right) \quad \text { and } \quad D_{s}^{\mathrm{CS}}(\phi)>D_{s}^{\mathrm{HdS}}(\phi) .
$$

We have never observed, in contrast to the findings in Refs. 8 and 13, that the peak in the $H(q)$ of charge-stabilized particles and the values of the associated short-time selfdiffusion coefficient are smaller than those of equal-density hard-sphere systems. Finally, we note that a reverse ordering relation, namely, $H^{\mathrm{CS}}(q \rightarrow 0)<H^{\mathrm{HdS}}(q \rightarrow 0)$, applies to the long-wavelength limiting form of $H(q){ }^{2,35,39}$ This reversed ordering relation in $H(q)$ near $q=0$ reflects the experimentally confirmed observation that charged particles sediment more slowly than uncharged ones (see, e.g., Ref. 40, for more details). The results on $H(q)$ included in Fig. 9 for sample LS-A exemplify this small- $q$ ordering relation (compare therein the solid and dashed-dotted lines).

\section{F. Discussion of earlier experiments: Does Hls screening occur?}

To comprehend the discussion on the controversy in the literature about the presence or absence of HIs screening in low-salt charge-stabilized systems, it is instructive to explore in some detail the concentration dependence of $H\left(q_{m}\right)$ as predicted by the $\delta \gamma$ theory. Curves for $H\left(q_{m}\right)$ vs $\phi$ are shown in Fig. 12(a). These curves are representative for de-ionized suspensions of particles with $a=50 \mathrm{~nm}$, similar to those studied in Ref. 8. It has been assumed in Fig. 12 that the effective charge is concentration independent.

As it is clearly seen, the curves for $H\left(q_{m}\right)$ are nonmonotonic in $\phi$ at higher particle charges. At small volume fractions, $H\left(q_{m}\right)$ is larger than the zero-density value of 1 . After passing through a maximum value larger than $1, H\left(q_{m}\right)$ sets in to decrease when $\phi$ is further increased. In contrast to this, the $H\left(q_{m}\right)$ of more weakly charged particles decreases monotonically below the zero-density value of 1 , similar to hardsphere suspensions. While the curves in Fig. 12(a) are based on the approximate $\delta \gamma$ theory with approximate RMSA in- 
puts for $S(q)$, all the qualitative features in $H\left(q_{m}\right)$ displayed in Fig. 12(a) are fully confirmed by our ASD simulations. The peak height of hard spheres is somewhat underestimated in the $\delta \gamma$ theory (for $\phi<0.35$ ), as can be noticed from Fig. 12(a). The system with $Z=10$ is practically hard-sphere-like [see Fig. 12(b)] which explains why the $\delta \gamma$ theory data for $H\left(q_{m}\right)$ are located a little below the hard-sphere curve given in Eq. (4). To be explicit we note that the $\delta \gamma$ theory predictions for hard spheres with Percus-Yevick input of $S(q)$ are well parametrized by the quadratic form $H\left(q_{m}\right)=1-2.03 \phi$ $+1.94 \phi^{2}$ for values of $\phi$ up to 0.5 .

Peak heights in $H(q)$ smaller than 1 that decrease with increasing volume fraction have been found experimentally in de-ionized suspensions in Refs. 41 and 42. In one of these papers ${ }^{41}$ this behavior was erroneously interpreted to be in contradiction to the expected one, i.e., to an $H\left(q_{m}\right)$ that should increase with volume fraction as observed for the more dilute samples studied in Refs. 43 and 44. In light of our discussion of Fig. 12, however, it can be realized that this conflict is only apparent: Whether $H\left(q_{m}\right)$ increases or decreases with concentration, and whether values larger or smaller than 1 are observed, depend crucially on the concentration range and on the particle interaction parameters. Figure 12(b) includes the RMSA calculations for the peak height, $S\left(q_{m}\right)$, of the static structure factor. It can be used to estimate roughly when the suspensions set in to freeze, as indicated by peak values in between 2.8 and 3.1 (HansenVerlet empirical freezing rule). For colloidal hard spheres, $S\left(q_{m}\right)$ is well parametrized for $\phi<0.5$ by the expression ${ }^{34}$

$$
S\left(q_{m}\right)=1+0.664 \phi \frac{1-0.5 \phi}{(1-\phi)^{3}} .
$$

The curve for the hard-sphere $S\left(q_{m}\right)$ is depicted in Fig. 12(b).

Riese et al. ${ }^{8}$ have argued that in de-ionized suspensions which are sufficiently concentrated, hydrodynamic screening of flow perturbations should be present as indicated by values of $H\left(q_{m}\right)$ smaller than the equal-density hard-sphere values. However, the experimental data for $H(q)$ presented in Ref. 8 are not self-consistent: the positions, $q_{m}$, of the peaks in $S(q)$ and in $D_{0} / D(q)$ as shown in Fig. 2 of Ref. 8, as well as the peak positions and the magnitudes both of the experimental and calculated curves for $H(q)$ depicted in Fig. 3 of Ref. 8 are not consistent with the particle radius and the system volume fraction provided by the authors. Moreover, the $H(q)$ shown in their Fig. 3 seems to be nonphysical, since the peak position of $H(q)$, which is equal to the peak position of $S(q)$, is located practically at the same wave number both for the de-ionized and for the equal-density hard-sphere system (system with $\phi=0.089$ ). This behavior is in conflict with other experimental, theoretical, and simulation work on lowsalt suspensions. What should be expected instead is that the peak position of the equal-density $S(q)$ and $H(q)$ of hard spheres is located at larger values of $q$ relative to those of the de-ionized system. A quantitative comparison of short-time diffusion results presented in Ref. 8 with our experimental data and with theoretical model calculations is not possible since, aside from the inconsistency in the peak position noted above, important system parameters are not provided in the paper, namely, the effective colloid charge, dielectric con- stant and the viscosity of the water/glycerol mixture (the latter two quantities depend significantly on temperature), the system temperature, and the value of $D_{0}$. Thus, the claim made in Ref. 8 that HIs screening should be present in deionized fluid suspensions of charged colloids and that, as a consequence, $\delta \gamma$ theory badly fails for these systems cannot be substantiated. It is not supported by other experimental data on $D(q)$ and $H(q)$. In fact, it conflicts to most other published data, and to the new results presented in this work.

In another publication, Haro-Perez et al. ${ }^{13}$ discussed backscattering dynamic light scattering measurements on charged liposomes. By probing the suspension dynamics at values of $q$ larger than the peak position of the static structure factor, these authors were able to estimate approximately the value of the short-time diffusion coefficient, $D_{s}$, for various liposome volume fractions. For the experiments performed at larger volume fractions, where the peak position in $S(q)$ is located on the right side of the experimentally selected $q$ value, the experimental findings were interpreted on the basis of low-density theoretical calculations of $H(q)$ using a RMSA input for $S(q)$, and compared to a second-order virial prediction for the hard-sphere value of $D_{s}$. From this analysis, the authors in Ref. 13 have extracted estimates for $D_{s}$ that are smaller than the equal-density hard-sphere values. With reference to the work in Ref. 8, it was concluded in Ref. 13 that effective screening of HIs should be operative for low-salt liposome solutions. A problematic point of the work in Ref. 13 is the way the volume fraction is calibrated: the peak position of the scattered light intensity, $I(q)$, was used to calculate the number concentration from the Bragg condition. The error in $\phi$ induced through such a procedure can be as large as $20 \%$. Since $\phi$ is a crucial parameter in this kind of analysis, the conclusions made in Ref. 13 regarding hydrodynamic screening are not decisive.

\section{CONCLUSIONS AND OUTLOOK}

We have described XPCS and SAXS measurements of the dynamic and static structure factors of charge-stabilized suspensions of fluorinated latex spheres at various concentrations and ionic strengths. Our experimental findings for the short-time diffusion properties embodied in $D(q)$ and $H(q)$ are in good agreement with (modified) $\delta \gamma$ theory calculations, and with accelerated Stokesian Dynamics simulation results, for all system parameters considered. The characteristics in the $H(q)$ for dense charge-stabilized suspensions are explainable by the effect of many-body HIs without any requirement to postulate hydrodynamic screening. These characteristics comprise, in particular, peak heights in $H(q)$ that can be smaller than 1 and, for de-ionized and dense systems, values of $H\left(q_{m}\right)$ that become smaller with increasing colloid concentration. As a generic short-time feature, our results show that the short-time self-diffusion coefficient and the peak height, $H\left(q_{m}\right)$, of charge-stabilized spheres are invariably larger than their corresponding hard-sphere values, provided the electrostatic screening length is nonzero. For fixed colloid concentration, both $D_{s}$ and $H\left(q_{m}\right)$ increase with decreasing salt content. Their largest values are attained in the limit of zero added salt. Several experimental and theoretical 
problems have been discussed with regard to the work in Refs. 8 and 13 that may be at the origin of the postulation of hydrodynamic screening. These problems must be overcome to obtain reliable data for $H(q)$ and $D_{s}$.

The present work has been focused on short-time diffusion properties. In the future, we shall study the long-time decay of $S(q, t)$ in charge-stabilized systems and the behavior of the associated long-time diffusion coefficients using both dynamic light scattering and XPCS. With regard to theory and simulation, we plan to investigate the long-time behavior of the dynamic structure factor using ASD simulations and mode coupling theory calculations. It will be interesting to explore whether the dynamic factorization behavior of the rate of change of $S(q, t)$, observed experimentally for hard spheres by Segre and Pusey, ${ }^{11}$ holds true also for chargestabilized particles. As a matter of fact, the question whether dynamic factorization is a generic feature even for hardsphere-like systems alone is not answered to data (see Ref. 45). A related theoretical prediction, made both for dense hard-sphere systems ${ }^{46}$ and dense charge-stabilized suspensions alike, ${ }^{47}$ is that $S(q, t)$ should decay exponentially at long times for wave numbers near to the location of the maximum of $S(q)$. While a long-time exponential decay near $q_{m}$ has been observed in DLS experiments on hard spheres, ${ }^{48}$ a similar extensive study has not been done so far for chargestabilized systems. We mention here that the time dependence of the XPCS dynamic scattering functions of fluorinated latex spheres at long times (not shown in this paper) is consistent with the predicted exponential long-time decay in $S\left(q_{m}, t\right)$. However, a more systematic study of long-time diffusion in charge-stabilized suspensions is required to allow for unequivocal conclusions.

\section{ACKNOWLEDGMENTS}

The authors are grateful to Stefano Saccana for providing them with excellent colloid samples. The authors appreciate the kind assistance of Andrei Fluerasu and Wolfgang Häussler in performing the XPCS experiments at the ESRF. This work has been supported by the Deutsche Forschungsgemeinschaft (SFB-TR6), by the Polish Ministry of Science and Higher Education (Project No. 1 PO3B 008 28), European Network of Excellence "Soft Matter Composites" (SoftComp No. S080118), and by CONICET and SECYTUNC, Argentina. One of the authors (A.J.B.) expresses his deep gratitude to Professor John Brady, who is a codeveloper of the ASD code for Brownian spheres. Without his contribution, the simulation results presented in this article would not have been possible.

${ }^{1}$ P. N. Pusey, in Liquids, Freezing and the Glass Transition, edited by J. P. Hansen, D. Levesque, and J. Zinn-Justin (Elsevier, Amsterdam, 1991).

${ }^{2}$ G. Nägele, Phys. Rep. 272, 215 (1996).

${ }^{3}$ A. J. C. Ladd, H. Gang, J. X. Zhu, and D. A. Weitz, Phys. Rev. E 52, 6550 (1995)

${ }^{4}$ A. J. Banchio, J. Gapinski, A. Patkowski, W. Häußler, A. Fluerasu, S. Sacanna, P. Holmqvist, G. Meier, M. P. Lettinga, and G. Nägele, Phys. Rev. Lett. 96, 138303 (2006).

${ }^{5}$ L. F. Rojas, R. Vavrin, C. Urban, J. Kohlbrecher, S. Stradner, F. Scheffold, and P. Schurtenberger, Faraday Discuss. 123, 385 (2003).

${ }^{6}$ M. Tirada-Miranda, C. Haro-Perez, M. Quesada-Perez, J. Callejas-
Fernandez, and R. Hidalgo-Alvarez, J. Colloid Interface Sci. 263, 74 (2003).

${ }^{7}$ E. Overbeck, C. Sinn, and M. Watzlawek, Phys. Rev. E 60, 1936 (1999).

${ }^{8}$ D. O. Riese, G. H. Wegdam, W. L. Vos, R. Sprik, D. Fenistein, J. H. H. Bongaerts, and G. Grübel, Phys. Rev. Lett. 85, 5460 (2000).

${ }^{9}$ D. Lumma, L. B. Lurio, M. A. Borthwick, P. Falus, and S. G. J. Mochrie, Phys. Rev. E 62, 8258 (2000).

${ }^{10}$ P. N. Pusey, P. N. Segre, O. P. Behrend, S. P. Meeker, and W. C. K. Poon, Physica A 235, 1 (1997).

${ }^{11}$ P. N. Segre and P. N. Pusey, Phys. Rev. Lett. 77, 771 (1996).

${ }^{12}$ J. Gapinski, A. Wilk, A. Patkowski, W. Häußler, A. J. Banchio, R. Pecora, and G. Nägele, J. Chem. Phys. 123, 054708 (2005).

${ }^{13}$ C. Haro-Perez, M. Quesada-Perez, J. Callejas-Fernandez, E. Casals, J. Estelrich, and R. Hidalgo-Alvarez, J. Chem. Phys. 119, 628 (2003).

${ }^{14}$ G. Petekidis, J. Gapinski, P. Seymour, J. S. van Duijneveldt, D. Vlassopoulos, and G. Fytas, Phys. Rev. E 69, 042401 (2004).

${ }^{15}$ S. Pyett and W. Richtering, J. Chem. Phys. 122, 034709 (2005).

${ }^{16}$ K. F. Seefeldt and M. J. Solomon, Phys. Rev. E 67, 050402 (2003).

${ }^{17}$ W. Härtl, Ch. Beck, and R. Hempelmann, J. Chem. Phys. 110, 7070 (1999).

${ }^{18}$ A. J. Banchio, G. Nägele, and J. Bergenholtz, J. Chem. Phys. 111, 8721 (1999).

${ }^{19}$ C. W. J. Beenakker and P. Mazur, Physica A 126A, 349 (1984).

${ }^{20}$ L. Durlofsky and J. F. Brady, Phys. Fluids 30, 3329 (1987).

${ }^{21}$ B. Cui, H. Diamant, B. Lin, and S. A. Rice, Phys. Rev. Lett. 92, 258301 (2004).

${ }^{22}$ B. Cui, H. Diamant, B. Lin, and S. A. Rice, Phys. Rev. Lett. 89, 188302 (2002).

${ }^{23}$ D. Long and A. Ajdari, Eur. Phys. J. E 4, 29 (2001).

${ }^{24}$ U. Genz and R. Klein, Physica A 171, 26 (1991).

${ }^{25}$ A. J. Banchio and J. F. Brady, J. Chem. Phys. 118, 10323 (2003).

${ }^{26}$ G. H. Koenderink, S. Sacanna, C. Pathmamanoharan, M. Rasa, and A. P. Philipse, Langmuir 17, 6086 (2001).

${ }^{27}$ T. Thurn-Albrecht, F. Zontone, G. Grübel, W. Steffen, P. MüllerBuschbaum, and A. Patkowski, Phys. Rev. E 68, 031407 (2003).

${ }^{28}$ W. B. Russel, D. A. Saville, and W. R. Schowalter, Colloidal Dispersions (Cambridge University Press, Cambridge, 1989).

${ }^{29}$ A. R. Denton, Phys. Rev. E 62, 3855 (2000).

${ }^{30}$ W. B. Russel and D. W. Benzing, J. Colloid Interface Sci. 83, 163 (1981).

${ }^{31}$ E. Trizac, L. Bocquet, M. Aubouy, and H. H. von Grünberg, Langmuir 19, 4027 (2003).

${ }^{32}$ F. J. Rogers and D. A. Young, Phys. Rev. A 30, 999 (1984).

${ }^{33}$ J. P. Hansen and J. B. Hayter, Mol. Phys. 46, 651 (1982).

${ }^{34}$ G. Nägele, The Physics of Colloidal Soft Matter, Lecture Notes Vol. 14 (Institute of Fundamental Technological Research, Polish Academy of Sciences, Warsaw, 2004).

${ }^{35}$ A. J. Banchio and G. Nägele (unpublished).

${ }^{36}$ A. J. C. Ladd, H. Gang, J. X. Zhu, and D. A. Weitz, Phys. Rev. E 52, 6550 (1995)

${ }^{37}$ B. Cichocki, M. L. Ekiel-Jezewska, and E. Wajnryb, J. Chem. Phys. 111, 3265 (1999)

${ }^{38}$ P. N. Segre, O. P. Behrend, and P. N. Pusey, Phys. Rev. E 52, 5070 (1995).

${ }^{39}$ M. Watzlawek and G. Nägele, J. Colloid Interface Sci. 214, 170 (1999).

${ }^{40}$ D. M. E. Thies-Weesie, A. P. Philipse, G. Nägele, B. Mandl, and R. Klein, J. Colloid Interface Sci. 176, 43 (1995).

${ }^{41}$ G. Grübel, D. Abernathy, D. O. Riese, W. L. Vos, and G. H. Wegdam, J. Appl. Crystallogr. 33, 424 (2000).

${ }^{42}$ D. O. Riese, W. L. Vos, G. H. Wegdam, F. J. Poelwijk, D. Abernathy, and G. Grübel, Phys. Rev. E 61, 1676 (2000).

${ }^{43}$ A. P. Philipse and A. Vrij, J. Chem. Phys. 88, 6459 (1988).

${ }^{44}$ J. K. Phalakornkul, A. P. Gast, R. Pecora, G. Nägele, A. Ferrante, B. Mandl-Steininger, and R. Klein, Phys. Rev. E 54, 661 (1996).

${ }^{45}$ L. B. Lurio, D. Lumma, A. R. Sandy, M. A. Borthwick, P. Falus, S. G. J. Mochrie, J. F. Pelletier, M. Sutton, A. Malik, and G. B. Stephenson, Phys. Rev. Lett. 84, 785 (2000).

${ }^{46}$ B. Cichocki and B. U. Felderhof, Physica A 204, 152 (1994).

${ }^{47}$ A. J. Banchio, G. Nägele, and J. Bergenholtz, J. Chem. Phys. 113, 3381 (2000).

${ }^{48}$ P. N. Segre, S. P. Meeker, P. N. Pusey, and W. C. K. Poon, Phys. Rev. Lett. 75, 958 (1995). 